\title{
A Novel Fairness-Aware Resource Allocation Scheme in Multiuser SISO-OFDMA Downlink
}

\author{
Vasileios D. Papoutsis, Ioannis G. Fraimis, and Stavros A. Kotsopoulos \\ Wireless Telecommunications Laboratory, Department of Electrical and Computer Engineering, University of Patras, \\ 26500 Rio, Greece \\ Correspondence should be addressed to Vasileios D. Papoutsis, vpapoutsis@ece.upatras.gr
}

Received 21 January 2010; Revised 11 May 2010; Accepted 21 June 2010

Academic Editor: Kwan L. Yeung

Copyright (๑) 2010 Vasileios D. Papoutsis et al. This is an open access article distributed under the Creative Commons Attribution License, which permits unrestricted use, distribution, and reproduction in any medium, provided the original work is properly cited.

\begin{abstract}
The problem of resource allocation for the downlink of wireless systems operating over a frequency-selective channel is investigated. It is assumed that both the Base Station (BS) and each user are equipped with a single antenna (Single Input Single Output-SISO case), and Orthogonal Frequency Division Multiple Access (OFDMA) is used as a multiple access scheme. The aim is to maximize the sum of the users' data rates subject to constraints on total available power and proportional fairness among users' data rates. Achieving the optimal solution has a high computational cost thereby the use of suboptimal techniques is necessary. A suboptimal, but efficient, scheme is devised, and it is shown, via simulation, that not only the proposed resource allocation scheme achieve higher sum of the users' data rates than other existing schemes but also the sum data rate is distributed fairly and flexibly among users. In addition, the proposed scheme is complexity effective and can be applied to latest-generation wireless systems that provide Quality-of-Service (QoS) guarantees.
\end{abstract}

\section{Introduction}

Orthogonal Frequency Division Multiplexing (OFDM) [1,2] has developed into a popular scheme for wideband wireless digital communication used in applications such as digital television and audio broadcasting, wireless networking and broadband internet access. The primary advantage of OFDM is its ability to cope with severe wireless channel conditions such as multipath fading which exists because of the frequency-selective nature of the wireless channel. Multipath fading leads to severe intersymbol interference (ISI) in both frequency and time. OFDM shows robustness against frequency-selective fading and tolerance to ISI.

As an extension, OFDM could be used not only as modulation scheme, but also as part of the OFDMA [3] scheme, which is also referred to as multiuser OFDM. OFDMA [4] is a multiuser version of the popular OFDM digital modulation scheme. In OFDMA, multiple access is achieved by first dividing the spectrum of interest into a number of subcarriers and then assigning subsets of the subcarriers to individual users. OFDMA helps exploit multiuser diversity in frequency-selective channels, since it is very likely that some subcarriers that are "bad" for a user are "good" for at least one of the other users $[5,6]$. Because of its superior performance in frequency-selective fading wireless channels, OFDMA is the modulation and multiple access scheme used in latest wireless systems such as IEEE 802.16e (Mobile WiMAX).

The optimality of the OFDMA network is proved in $[7,8]$ where it is shown that the optimal policy is that each subcarrier be exclusively assigned to only one user, eliminating the intracell interference (ICI). In [7], the optimality of OFDMA is proved in a downlink multiuser OFDM system with adaptive Quadrature Amplitude Modulation (QAM) and independent decoding. However, the proof cannot be generalized to other modulation schemes. This generalization is shown in [8] where it is proved that the optimality of OFDMA holds for any adaptive modulation scheme which can be approximated using a convex data rateSNR/SINR function [9].

There are two approaches to allocate subcarriers: fixed and adaptive allocations. Fixed allocations use time division 
multiple access (TDMA) or frequency division multiple access (FDMA) as multiaccess schemes to allocate each user a predetermined time slot or frequency band for transmission. While applying fixed allocation, the system neglects the channel diversity and does not use the deeply faded subcarriers for other users which do not seem as deeply faded to them. In [10], these two fixed allocation schemes are discussed and compared in much detail. On the other hand, adaptive allocations allocate resources to users based on their channel gains. Due to the time-varying nature of the wireless channel, dynamic resource allocation makes full use of the multiuser diversity to achieve higher performance.

In recent years, many dynamic resource allocation schemes for the SISO-OFDMA systems have been developed to find the solution of either minimizing the overall transmit power [11-16], given constraints on the users' data rates, or Bit Error Rate (BER) or maximizing the sum of the users' data rates $[7,17-26]$, with a total transmit power constraint. The former is called Margin Adaptive (MA) problem, and the latter is called Rate Adaptive (RA) problem [27].

The MA problem has been less investigated than the RA problem. In [11], total transmit power is minimized by assigning, to each user, a set of subcarriers within an OFDM symbol and by determining, for each subcarrier, the number of bits to be transmitted and the power level used. A suboptimal iterative algorithm based on the Lagrangian relaxation method is presented. In [12], the work of [11] is extended by developing a low complexity algorithm to solve the optimization problem without performance degradation. In [13], the concept of marginal utility is developed, and the subcarrier with the largest marginal utility is assigned to its corresponding user at each subcarrier allocation iteration. In [14], the total power consumption is minimized with constraints on BER and transmission data rate for users requiring different classes of service. In [15], a heuristic algorithm based on constructive assignment and iterative improvement is proposed which can give out a valid solution in real time. Finally, in [16], in order to minimize the total transmit power with proportional data rate constraints, the nonlinear optimization is converted into linear optimization, and the complexity of the proposed bit and power allocation algorithm is considerably reduced.

Many algorithms have been proposed to solve the RA problem. In [7], it is proved that the sum of the users' data rates is maximized when each subcarrier is assigned to the user with the best subcarrier gain, and power is then distributed by the water-filling algorithm [28, 29]. This strategy followed by the use of adaptive modulation [30] is known to be optimal. However, an inherent drawback of the maximum sum capacity criterion is the lack of fairness, because certain users may be completely shut off during a scheduling period. This is dealt with by imposing fairness criteria, such as the proportional data rate constraint considered in this paper. In [17], fairness in the resource allocation is incorporated through maximizing the minimum data rate among users. In $[18,19]$, a proportional fairness criterion, that is determined by the system QoS guarantees, is employed. In [18], an iterative method for root finding of nonlinear equation is used which is complex and time consuming. In [19], a low complexity noniterative data rate adaptive resource allocation scheme is proposed that linearizes the power allocation problem and yields higher sum of the users' data rates than [18]. The algorithms of $[18,19]$ impose a ratio $\left(\gamma_{1}: \cdots: \gamma_{k}\right)$ among the data rates of the users that is determined by the system QoS guarantees. By setting ( $\gamma_{1}$ : $\left.\cdots: \gamma_{k}=1: \cdots: 1\right)$, the proportional fairness criterion reduces to that of [17]. In [20], the subcarrier allocation algorithm is based on prioritizing the most sensitive user in the system, and the variance of the subcarrier gains for each user is used to define the sensitivity of the user to the subcarrier allocation. In [21], a joint subcarrier and power allocation algorithm is proposed which is based on [17]. In $[22,23]$, the fulfillment of every user's data rate constraints is guaranteed, and in [24], the proposed algorithms ensure a fair resource allocation in terms of the number of subcarriers with affordable data rates. However, in [24], the proportional data rate constraint is not applied, and an equal number of subcarriers is assigned to each user with the magnitude of the channel frequency responses being the only factor for determining the subcarrier allocation. In [25], the sum of the users' data rates is maximized with long-term access proportional fairness which means that every user has the same average channel access probability, and proportional data rate constraint is not applied too. Finally, in [26], the sum of the users' data rates is maximized but the resource allocation unit is not the subcarrier, as in previous algorithms $[7,11-25]$, but a time/frequency unit (slot), in accordance with WiMAX systems.

In this paper, the resource allocation problem is investigated. We concentrate more on maximizing both the sum of the users' data rates and data rate fairness among the users. The objective is to maximize the sum of the users' data rates beng subjected to constraints on the total available power and proportional data rates among users. The proposed method, which is also complexity effective, consists of three algorithms: an algorithm that determines the number of subcarriers for each user, a subcarrier allocation algorithm by dividing the users in two groups, and the water-filling algorithm [28, 29]. The first two algorithms assign the available subcarriers to the users of the system, and the third one allocates the available power optimally.

The remainder of the paper is organized as follows. The problem of sum data rate maximization using proportional data rate constraints is formulated in Section 2. The proposed scheme introduced in Sections 3 and 4 contains the complexity analysis of the proposed algorithm and a complexity comparison with other algorithms. Simulation results and a comparison between the proposed scheme and other existing schemes are provided in Section 5. Finally, Section 6 contains concluding remarks.

\section{System Model and Problem Formulation}

The block diagram of a SISO-OFDMA downlink system model is shown in Figure 1. The following assumptions are used in this paper: (i) the time-varying channels between the BS and different users are assumed to be frequencyselective wireless channels with independent Rayleigh fading, 


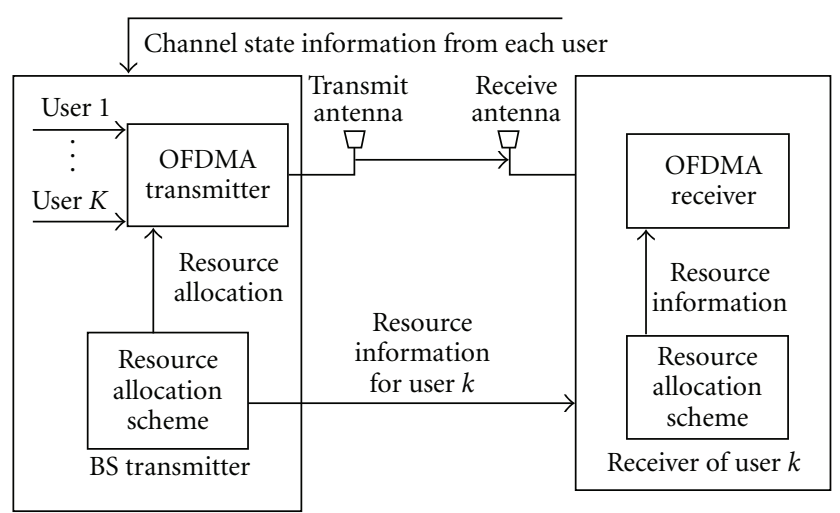

FIGURE 1: SISO-OFDMA downlink block diagram.

and the channel can be regarded as constant during the resource allocation period; (ii) the ISI is completely removed by exploiting OFDM techniques; that is, the width of each subcarrier is much smaller than the coherence bandwidth of the channel. Thus, each user experiences flat fading on each subcarrier; (iii) the Channel State Information (CSI) is perfectly known by the receiver, and each user feedbacks a certain form of channel information correctly to the BS; (iv) each subcarrier can be used only by one user at each time $[7,8]$.

Figure 1 shows that in the BS, CSI is sent to the resource allocation scheme block through the feedback channels. The resource allocation scheme is forwarded to the SISO-OFDMA transmitter. The transmitter then forms the transmitted OFDMA symbol by selecting different number of bits from different users. The resource allocation scheme is updated as soon as the channel information is collected and also the resource allocation information is sent to each user for detecting.

Consider an OFDMA downlink transmission in a single cell with $K$ active users and $N$ subcarriers. $P_{\text {tot }}$ is the total transmit power, and the channel gain for user $k$ on subcarrier $n$ is denoted by $g_{k, n}$. Each subcarrier $n$ of user $k$ is assigned a power $p_{k, n}$. With the noise power spectral density being $N_{0}$ and the total bandwidth of all subcarriers being $B$, the additive white noise power is $\sigma^{2}=N_{0} B / N$. Therefore, the subcarrier SNR can be expressed as $h_{k, n}=g_{k, n}^{2} / \sigma^{2}$ and the received SNR of user $k$ on subcarrier $n$ is $\gamma_{k, n}=p_{k, n} h_{k, n}$.

Each of the user's bits is modulated into $N$ M-level QAM symbols, which are subsequently combined using the IFFT into an OFDMA symbol. For a square M-level QAM using Gray bit mapping as a function of received SNR $\gamma_{k, n}$ and number of bits of user $k$ on subcarrier $n r_{k, n}$, the BER can be approximated to within $1 \mathrm{~dB}$ for $r_{k, n} \geq 4$ and $\mathrm{BER} \leq 10^{-3}$ as in [31]

$$
\operatorname{BER}_{\text {MQAM }}\left(\gamma_{k, n}\right) \approx \frac{1}{5} \exp \left[\frac{-1.6 \gamma_{k, n}}{2^{r_{k, n}}-1}\right] .
$$

By solving (1), $r_{k, n}$ is

$$
r_{k, n}=\log _{2}\left(1+\frac{\gamma_{k, n}}{\Gamma}\right)=\log _{2}\left(1+p_{k, n} H_{k, n}\right),
$$

where $\Gamma=-\ln (5 \mathrm{BER}) / 1.6$, and $H_{k, n}=h_{k, n} / \Gamma$ is the effective subcarrier SNR of user $k$ on subcarrier $n$.

By applying the conclusions above, the optimization problem can be formulated as

$$
\max _{c_{k, n}, p} \frac{B}{K} \sum_{k=1}^{K} \sum_{n=1}^{N} c_{k, n} r_{k, n}
$$

subject to

$$
\begin{gathered}
\mathcal{c}_{k, n} \in\{0,1\}, \quad \forall k, n, \\
p_{k, n} \geq 0, \quad \forall k, n, \\
\sum_{k=1}^{K} c_{k, n}=1, \quad \forall n, \\
\sum_{k=1}^{K} \sum_{n=1}^{N} c_{k, n} p_{k, n} \leq P_{\text {tot }}, \\
R_{1}: R_{2}: \cdots: R_{K}=\gamma_{1}: \gamma_{2}: \cdots: \gamma_{K},
\end{gathered}
$$

where $c_{k, n}$ is the subcarrier allocation indicator such that $c_{k, n}=1$ if subcarrier $n$ is assigned to user $k$ and $c_{k, n}=0$ if not. Constraints (4) ensure the correct values for the subcarrier allocation indicator and the power, respectively. Constraint (5) restricts the assignment of each subcarrier to only one user, and (6) is the power constraint. The total data rate for user $k$, denoted as $R_{k}$, is defined as

$$
R_{k}=\frac{B}{N} \sum_{n=1}^{N} c_{k, n} r_{k, n}
$$

where $r_{k, n}$ is given by (2), and $\left\{\gamma_{k}\right\}_{k=1}^{K}$ in (7) are the proportional data rate constraints.

Note that problem (3) is an NP-hard combinatorial optimization problem [32] with nonlinear constraints. In a system with $K$ users and $N$ subcarriers, there are $K^{N}$ possible subcarrier assignments, since it is assumed that no subcarrier can be used by more than one user. For a certain subcarrier assignment, an optimal power distribution based on water-filling $[28,29]$ can be used to maximize the sum of the users' data rates, while maintaining proportional fairness. The maximum sum data rate over all $K^{N}$ subcarrier assignment schemes is the global maximum, and the corresponding subcarrier assignment and power distribution are the optimal resource allocation scheme. However, it is difficult to obtain an optimal solution within any reasonable time frame. As a result, a novel and cost-effective resource allocation scheme is formulated to solve this problem.

\section{The Proposed Resource Allocation Scheme}

Ideally, power and subcarriers should be allocated jointly to solve optimization problem (3) optimally. This process has a prohibitive computational complexity. Proposed RA schemes such as in $[7,18,19]$ handle subcarrier and power allocation separately but the complexity of the power allocation is very high. Exception is the proposed algorithm in [21] 
where joint subcarrier and power allocation is performed. Again, the complexity is very high, and the solution is not optimal because not only is it found by alternating between subcarrier and power allocation, but also in the first step of this algorithm, equal power allocation scheme is adopted. On the other hand, algorithms such as [17, 20, 22, 24] use fixed power allocation, that is, uniform power distribution among available subcarriers and perform only subcarrier allocation. In the following, a suboptimal resource allocation scheme is proposed which consists of three algorithms and assures a low complexity performance.

(A1) Determine the number of subcarriers $N_{k}$ to be initially assigned to each user.

(A2) Assign the subcarriers to each user in a way that the sum of the users' data rates is maximized while maintaining rough proportionality among users' data rates.

(A3) Allocate the available power $P_{\text {tot }}$ to assigned subcarriers $N_{k}$ using the water-filling $[28,29]$ algorithm.

3.1. (A1) Number of Subcarriers of Each User. In this algorithm, the number of subcarriers $N_{k}$ for each user is determined. This process is based on the average effective subcarrier SNR of each user which is calculated by

$$
\bar{H}_{k}=\frac{1}{N} \sum_{n=1}^{N} H_{k, n}, \quad \forall k=1,2, \ldots, K .
$$

The approximate data rate of each user is

$$
\bar{R}_{k}=N_{k} \frac{B}{N} \log _{2}\left(1+\bar{H}_{k} \bar{P}\right), \quad \forall k=1,2, \ldots, K,
$$

where $\bar{P}$ is the equal power allocation among subcarriers. At each iteration, the user with the lowest ratio $\bar{R}_{k} / \gamma_{k}$ has the option to be assigned one more subcarrier. When all the available subcarriers are assigned to $K$ users of the system, the approximate number of subcarriers $N_{k}$ for each user is gotten. This algorithm is as follows.

(1) Initialization:

(i) Set $\left\{\gamma_{k}\right\}_{k=1}^{K}$, the proportional data rate constraints.

(ii) Set the initial number of subcarriers $N_{k}=$ $\left\lfloor N\left(\gamma_{k} / \sum_{k=1}^{K} \gamma_{k}\right)\right\rfloor$, for all $k=1,2, \ldots, K$ and $N_{\mathrm{al}}=\sum_{k=1}^{K} N_{k}$.

(iii) Get the average effective subcarrier SNR for each user using (9).

(2) Approximate data rate of each user of initialization:

(i) Get the equal power to each allocated subcarrier $\bar{P}=P_{\text {tot }} / N_{\text {al }}$.

(ii) Calculate $\bar{R}_{k}$, for all $k=1,2, \ldots, K$, using (10).
(3) While $N_{\text {al }} \leq N$ :

(i) Find $k$ satisfying $\bar{R}_{k} / \gamma_{k} \leq \bar{R}_{i} / \gamma_{i}$, for all $i, 1 \leq$ $i \leq K$. For the found $k$, let $N_{k}=N_{k}+1$ and $N_{\mathrm{al}}=N_{\mathrm{al}}+1$.

(ii) Get the equal power to each allocated subcarrier $\bar{P}=P_{\text {tot }} / N_{\mathrm{al}}$.

(iii) Calculate $\bar{R}_{k}$, for all $k=1,2, \ldots, K$, using (10).

In initialization step, $N\left(\gamma_{k} / \sum_{k=1}^{K} \gamma_{k}\right)$ is approximated to the lower integer because $N_{k}$ should be an integer. Hence, it is not sure that $N_{\mathrm{al}}=N$; there might be some remaining subcarriers. That is the reason why step (3) of the algorithm is necessary.

In the next subsection, the subcarrier assignment algorithm is introduced.

3.2. (A2) Subcarrier Assignment to Available Users. In this subsection, the $N_{k}$, for all $k=1,2, \ldots, K$ subcarriers are allocated to available users in order to maximize the sum of the users' data rates while maintaining rough proportionality among the data rates of $K$ users. The algorithm is described below.

(1) Initialization:

(i) Set $A=\{1,2, \ldots, N\}, R_{k}=0$, for all $k=$ $1,2, \ldots K, c_{k, n}=0$, for all $k=1,2, \ldots, K$ and $n \in A$.

(ii) Sort the $K$ users by average effective subcarrier SNR, that is, $\bar{H}_{1} \leq \cdots \leq \bar{H}_{m} \leq \cdots \leq \bar{H}_{K}$ without loss of generality.

(iii) Divide the $K$ users into two groups:

(a) Bad effective subcarrier SNR group: user $_{\mathrm{b}}=\{1,2, \ldots, m\}$.

(b) Good effective subcarrier SNR group: user $_{\mathrm{g}}=\{m+1, m+2, \ldots, K\}$.

(2) For $k=1,2, \ldots, m$ :

(i) Find $n$ satisfying $H_{k, n} \geq H_{k, j}$, for all $j \in A$.

(ii) For the found $n$, set $c_{k, n}=1, N_{k}=N_{k}-1, A=$ $A-\{n\}$ and update $R_{k}$ according to (8). In (8), $p_{k, n}=P_{\text {tot }} / N$.

(3) While $\mid$ user $_{\mathrm{b}} \mid \neq \varnothing$ :

(i) Find $k$ satisfying $R_{k} / \gamma_{k} \leq R_{i} / \gamma_{i}$, for all $i \in$ user .

(ii) For the found $k$, if $N_{k}>0$

(a) Find $n$ satisfying $H_{k, n} \geq H_{k, j}$, for all $j \in$ A.

(b) Set $c_{k, n}=1, N_{k}=N_{k}-1, A=A-\{n\}$ and update $R_{k}$ according to (8). In (8), $p_{k, n}=$ $P_{\text {tot }} / \mathrm{N}$.

(iii) else
(a) user $_{\mathrm{b}}=$ user $_{\mathrm{b}}-\{k\}$.

(3) Redo:

(i) Steps (2) and (3) for the good effective subcarrier SNR group, that is, for users $\in$ userg $_{\text {g }}$. 
In step (1) of the subcarrier assignment to available users algorithm, all the variables are initialized. $A$ is the set of available subcarriers; $c_{k, n}$ is the subcarrier allocation indicator; $R_{k}$ is a vector which keeps track of the data rate of each user $k=1,2, \ldots, K$. Then, users are divided in two groups according to parameter $m$ : the user $\mathrm{b}_{\mathrm{b}}$ and the user ${ }_{g}$, the group of users with bad average effective subcarrier SNR and the group with good average effective subcarrier SNR, respectively. Parameter $m$ is chosen in such a way that the two user groups contain the same number of users if $K$ is an even number. Otherwise, if $K$ is an odd number, one of the two user groups would contain one more user than the other group.

In step (2), each user of userb group is assigned the available subcarrier on which he/she has the largest effective subcarrier SNR. Note that an inherent advantage is gained by the fact that users of user group choose their best subcarrier earlier than the users of the other group.

In step (3), subcarriers are assigned to available users until each user gets his allotment of $N_{k}$ subcarriers. The user who has the least data rate divided by its proportional constant has the priority to choose his best subcarrier. The best subcarrier is that on which he has the largest effective subcarrier SNR. The user, who gets his allotment of $N_{k}$ subcarriers, can no longer be assigned any more subcarriers. $\mid$ user $_{b} \mid$ here denotes the cardinality of set user $_{\mathrm{b}}$.

In step (4), the same procedure takes place but for the user $_{\text {g }}$ group; the group of users with good average effective subcarrier SNR. The condition $k=1,2, \ldots, m$, changes to $k=m+1, m+2, \ldots, K$.

3.3. (A3) Power Allocation. Subcarrier assignment to available users algorithm is suboptimal because available power is distributed uniformly among subcarriers. So the subcarrier allocation is kept, but the available power $P_{\text {tot }}$ is assigned to subcarriers using the water-filling algorithm $[28,29]$ :

$$
p_{k, n}=\left(\lambda-\frac{1}{H_{k, n}}\right)^{+}, \quad \forall k, n,
$$

where $p_{k, n}$ is the allocated power in each subcarrier, $(\cdot)^{+}=$ $\max (0, \cdot)$, and $\lambda$ satisfies

$$
\sum_{k=1}^{K} \sum_{n=1}^{N} p_{k, n}=P_{\text {tot }}
$$

\section{Complexity Analysis}

In order to analyze the computational complexity of the proposed scheme and compare it with other existing algorithms, recall that $K$ refers to the total number of users in the system, and $N$ refers to the number of subcarriers, which is much larger than $K$.

Initialization step of the first algorithm of the proposed scheme requires $K$ multiplications to set the initial number of subcarriers $N_{k}$, and also average effective subcarrier SNR is calculated $K$ times. Thus, the complexity of this initialization step is $O(K)$. In second step of the same algorithm, $\bar{R}_{k}$ is calculated $K$ times which is $O(K)$. In third step, the user with the least $\bar{R}_{k} / \gamma_{k}$ among $K$ users is found and $\bar{R}_{k}$ is calculated for $k=1,2, \ldots, K$. This is repeated until $N_{\mathrm{al}}=N$. Thus, this step requires $O\left(K\left(N-N_{\mathrm{al}}\right)\right)$ complexity which is also the overall complexity of the first algorithm of the proposed resource allocation scheme.

In initialization step of the second algorithm of the proposed resource allocation scheme, $K$ users are sorted by average effective subcarrier SNR which has $O\left(K \log _{2} K\right)$ complexity. Then, the complexity of the division of $K$ users in two groups is $O(K)$. Thus, the complexity of this initialization step is $O\left(K \log _{2} K\right)$. In the second step, for each user of one group, the best subcarrier is found which has complexity $O(K N)$ because in our simulations the two groups contain equal number of users, that is, $m=K / 2$. In step three of this algorithm, subcarriers are allocated to users of one group until each user gets his allotment of $N_{k}$ subcarriers. In the worst case scenario, the complexity of this step is $O(K N)$. In step four, the same procedure takes place but for the other group of users. Thus, because $K \ll N$ and $\log _{2} K \ll N$, the complexity of the second algorithm of the proposed resource allocation scheme is $O(K N)$.

Finally, in the third algorithm of the proposed resource allocation scheme, the water-filling power allocation algorithm is implemented which requires finding $\lambda$. The update of $\lambda$ can be done by using a simple bisection method until the sum power converges [9].

In the resource allocation algorithm proposed in [7], each subcarrier is allocated to the user which has the best channel gain on it, and then water-filling power allocation is applied to subcarriers. Hence, the complexity for the subcarrier allocation is $O(K N)$ which is the same as the complexity of the proposed resource allocation scheme which also takes fairness into account.

Algorithms proposed in $[18,19]$ consist of similar subcarrier allocation steps but differ in power allocation procedure. The algorithm proposed in [18] has a stage of subcarrier allocation with a complexity of $O(K N)$ followed by a second stage of power allocation, which requires an iterative method to solve $K$ nonlinear variables followed by the water-filling to be performed $K$ times. The complexity of each iteration has been shown to be $O(K)$ [18]. Furthermore, the existence of the power allocation scheme for each user should be checked [18] before performing water-filling algorithm for each user. The algorithm proposed in [19] consists of four steps. As described in [19], the complexity of the first two steps are $O(K)$ and $O\left(K N \log _{2} N\right)$, respectively. The third step of power allocation requires simple operations which are significantly less complex than the power allocation step of [18]. Furthermore, the power allocation step of [18] also needs a high subchannel SNR assumption to function properly, for which the power allocation step of [19] does not make. Consequently, the real computational savings of [19] compared to [18] can be seen in the power allocation step. The complexity of the proposed resource allocation scheme is less than that of the algorithms proposed in $[18,19]$. 
The algorithm proposed in [20] consists of two steps. The first step has a complexity of $O(K)$. Then, $N$ subcarriers are sorted for $\mathrm{K}$ users with a complexity of $\mathrm{O}\left(\mathrm{KN} \log _{2} \mathrm{~N}\right)$. The complexity of the second step is $O(K N)$. Therefore, the overall complexity is $O\left(K N \log _{2} N\right)$ which is a bit larger compared to the complexity of the proposed resource allocation scheme. However, the proposed resource allocation scheme uses the water-filling algorithm which is more complex than the equal power allocation scheme used in [20] but, as said earlier, it can be easily implemented by using a simple bisection method [9] to find the appropriate $\lambda$. Moreover, simulation results in Section 5 show the real advantages of the proposed resource allocation scheme compared to that of [20].

In the algorithm proposed in [21], optimization of the $K+N$ parameters is carried out by alternating between subcarrier and power allocation. The water-filling power allocation is used for each user. However, unlike the previous approaches, water-filling for each user plays a crucial role in deciding the subcarrier allocation. When a subcarrier is allocated to a user, the power allocated to the user is incremented by $P_{\text {tot }} / N$, that is, the power allocated to each user is proportional to the number of subcarriers currently allocated to the user. The user's data rate is also updated assuming that water filling is used. This updated data rate information is used in the allocation of the remaining subcarriers. Thus, the complexity of this algorithm, without taking into account the water filling, is $O(K N)$ which is the same as the complexity of the resource allocation scheme proposed in this paper.

\section{Simulation Results}

In this section, the performance of the proposed resource allocation scheme is evaluated using simulation. In all simulations presented in this section, the frequency-selective channel consists of six independent Rayleigh multipath components (taps). Each multipath component is generated using the Jakes model [33]. As in [18], an exponentially decaying power delay profile is assumed, the ratio of the energy of the $l$ th tap to the first tap being equal to $e^{-2 l}$. For each channel realization, the proposed scheme is used to perform resource allocation, and the data rates of each user are computed. A maximum delay spread of $5 \mu \mathrm{s}$ and maximum doppler of $30 \mathrm{~Hz}$ are assumed. The channel information is sampled every $0.5 \mathrm{~ms}$ to update the resource allocation. As in $[18,19]$, the total available bandwidth is equal to $B=$ $1 \mathrm{MHz}$, and the total transmitted power of the BS is equal to $P_{\text {tot }}=1 \mathrm{~W}$. The number of subcarriers of an OFDM symbol is $N=64$, and variance of the additive noise is equal to $N_{0}=-80 \mathrm{~dB} \cdot \mathrm{W} / \mathrm{Hz}$ (single-sided PSD). The BER $=10^{-7}$, and the average subchannel SNR $=10 \log \left(P_{\text {tot }} /\left(B N_{0} / N\right)\right)$ is assumed to be $38 \mathrm{~dB}$. The number of channel realizations is equal to 1000 , and 100 time samples for each realization are used for each user. Parameter $m$ is equal to $m=K / 2$.

For each channel realization, a set of proportional constants are assigned to each user. It is assumed that these

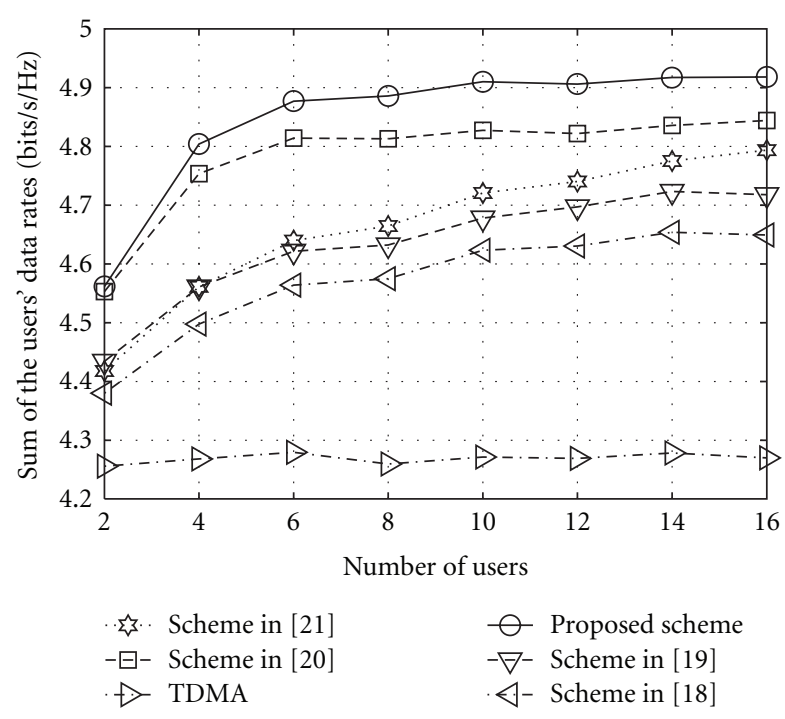

Figure 2: Sum of the users' data rates.

constants follow the probability mass function

$$
p_{\gamma_{k}}= \begin{cases}1, & \text { with probability } 0.5 \\ 2, & \text { with probability } 0.3 \\ 4, & \text { with probability } 0.2\end{cases}
$$

In Figures 2-6, the number of users for the system varies from 2 to 16 in increment of 2 . Figures 2, 3, and 4 depict the comparison of the sum of the users' data rates and normalized data rate ratios per user, respectively, between the proposed method, the methods proposed in [18-21], and a static TDMA scheme. It is demonstrated that under the same conditions, the proposed resource allocation scheme achieves much higher sum of users' data rates compared with the aforementioned methods. Because all these schemes use the same simulation parameters, such great improvement in sum of users' data rates is brought solely by the priority scheduling (division of users in two groups) introduced in Section 3 which exploits more efficiently the multiuser diversity. In order to be more clear, the comparison of normalized data rate ratios is shown in two figures: Figures 3 and 4 . In these figures, the normalized data rate proportions are given by $R_{k} / \sum_{k=1}^{K} R_{k}$, for all $k=$ $1,2, \ldots, K$ and Gamma means the strict data rate proportions specified by $\gamma_{k} / \sum_{k=1}^{K} \gamma_{k}$, for all $k=1,2, \ldots, K$, averaged over 100 channels when all users are present in the system. It is observed that each method obtains a tradeoff between sum of the users' data rates and relaxed proportionality among users. The proposed method distributes the sum data rate very well among users, very close to the defined ideal data rate constraints. In addition, what we are most concerned with is the sum of the users' data rates and rough data rate proportions, close to the ideal, among users are acceptable in practical systems.

Figures 5 and 6 depict the comparison of the sum of the users' data rates gain over TDMA between the proposed 


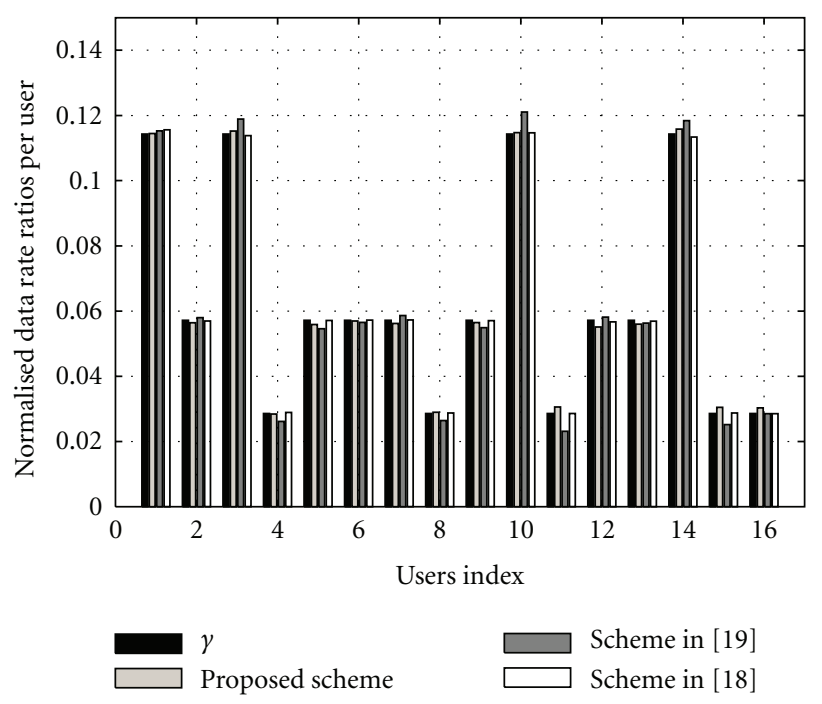

Figure 3: Normalized data rate ratios per user.

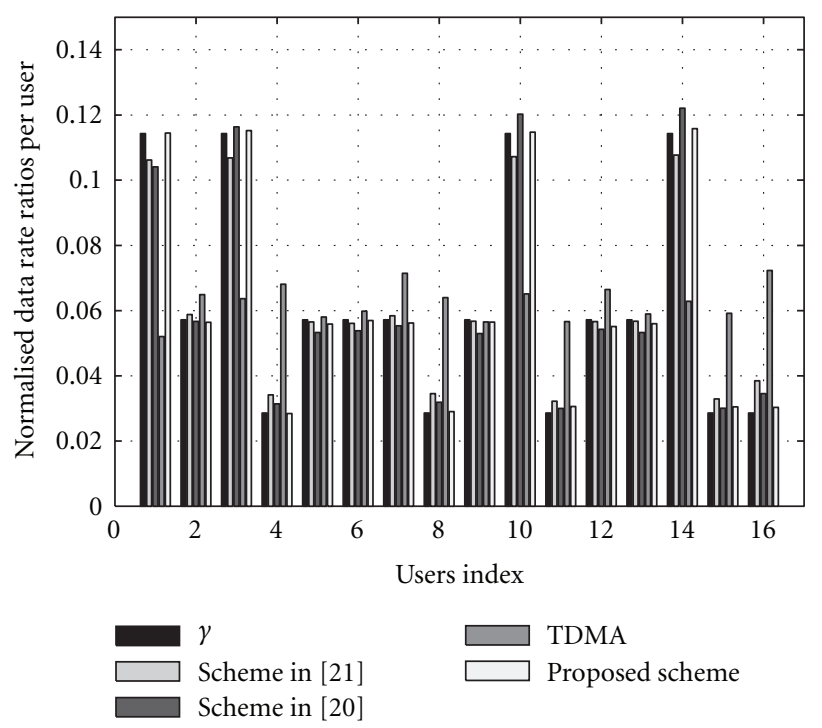

FIGURE 4: Normalized data rate ratios per user.

method and the methods proposed in [18-21] in bits/s/Hz and in percentage, respectively. It is observed that the more users in the system, the higher the gain these methods can achieve. The proposed method achieves the higher gain among the others which could be explained by the fact that it exploits better the multiuser diversity taking into account the innovative priority scheduling. Moreover, the more users in the system, the lower the probability that a subcarrier is in a deep fading to all the available users.

In Figures 7-12, the system consists of $K=8$ users. It is assumed that the average channel gain for the first user is $10 \mathrm{~dB}$ higher than that of the other users. The different fairness constraints are $\gamma_{1}=2^{p}$ and $\gamma_{2}=\gamma_{3}=\cdots=$ $\gamma_{8}=1$ for $p=0,1,2, \ldots, 7$. Thus, there are 8 sets of fairness constraints. These figures depict the comparison between the

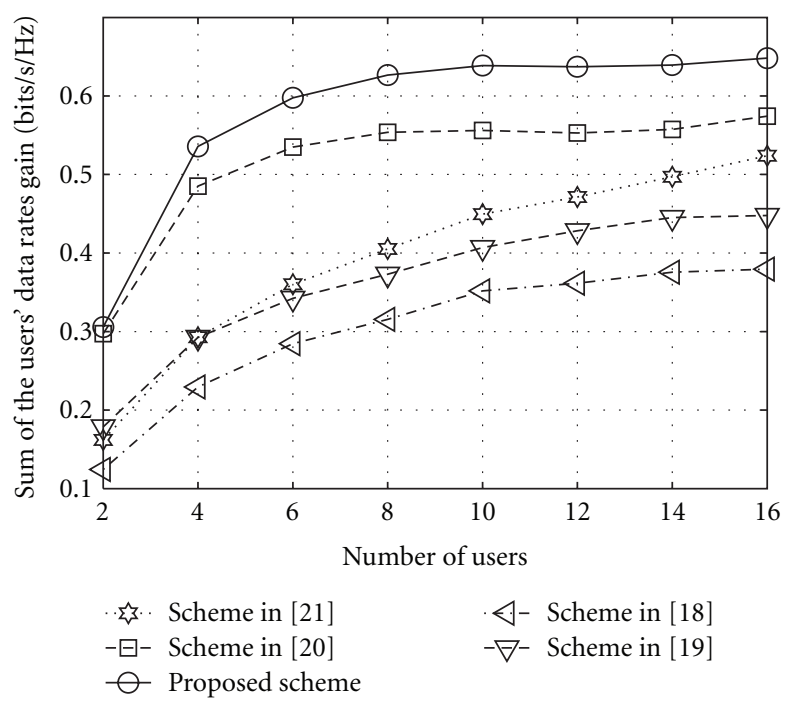

Figure 5: Sum of the users' data rates gain.

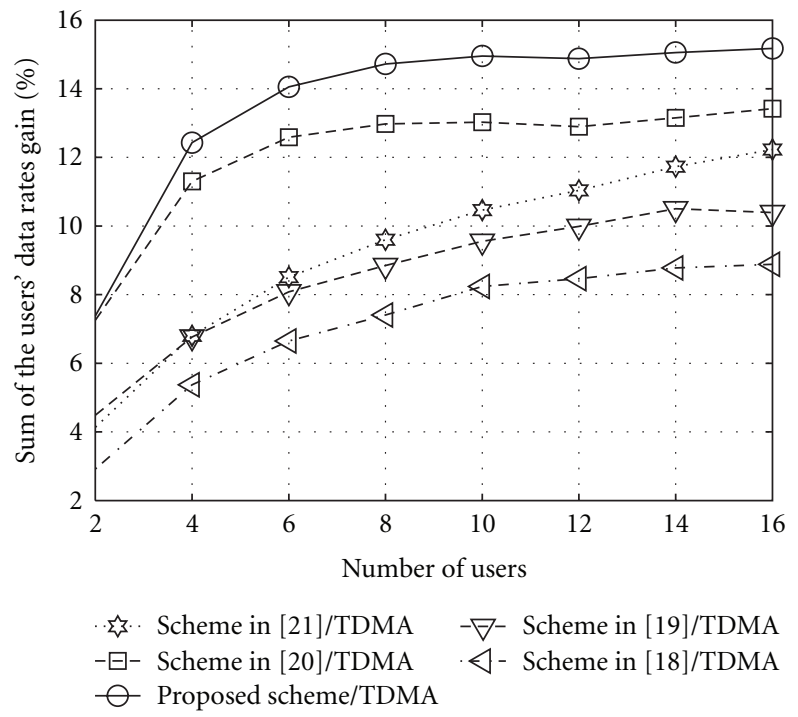

FIgURE 6: Sum of the users' data rates gain (\%).

proposed resource allocation scheme, the schemes proposed in [18-21], and a static TDMA scheme.

In Figures 7 and 8, the sum of the users' data rates versus different fairness constraints is depicted. The proposed resource allocation scheme achieves higher sum of the users' data rates than the other schemes. As is expected, the resulting sum of the users' data rates is smaller compared to the one achieved by the algorithm proposed in [7], since the latter is not constrained by fairness requirements, and all resources are allocated to the users with the best channel gains. The sum of the users' data rates achieved by the proposed scheme varies as the data rate constraints change. As more priority is allocated to user 1, that is, as the index $p$ increases, higher total sum of the users' data rates is achieved which is reasonable since user 1 has higher average channel gain and hence 


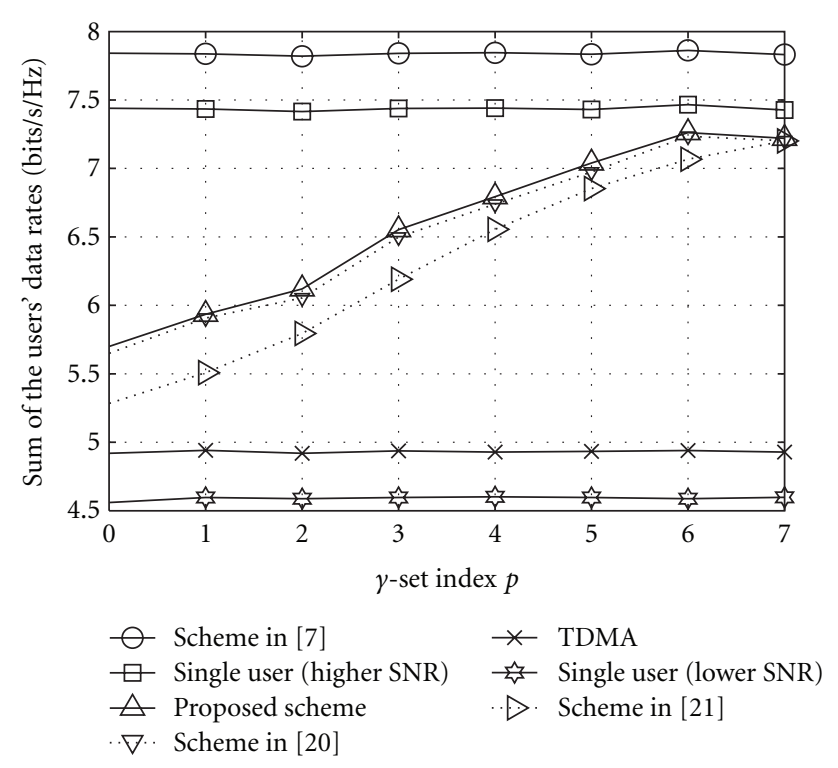

Figure 7: Sum of the users' data rates.

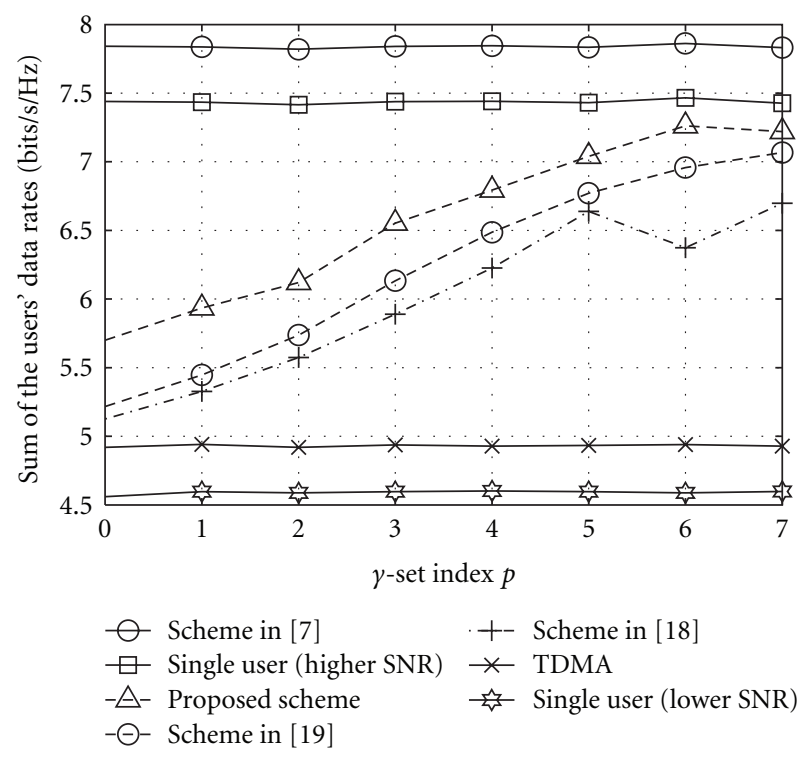

Figure 8: Sum of the users' data rates.

can more efficiently utilize the resources. It is also shown that the sum of the users' data rates of the method in [7] and static TDMA is the same by varying the gamma set values, because there is no fairness guarantee mechanism in these systems. The users' data rates of two types of single-user systems are also shown: one for the user with high average channel gain and the other for the user with low average channel gain.

Figures $9,10,11$, and 12 depict the normalized data rate ratios of each user for gamma-set indices $p=0$ and $p=3$. For $p=0, \gamma_{1}=2^{0}=1$, and $\gamma_{2}=\gamma_{3}=\cdots=\gamma_{8}=1$ while, for $p=3, \gamma_{1}=2^{3}=8$, and $\gamma_{2}=\gamma_{3}=\cdots=\gamma_{8}=1$. It is shown that for the method proposed in [7], user 1 gets most of the available subcarriers and occupies almost all of the sum

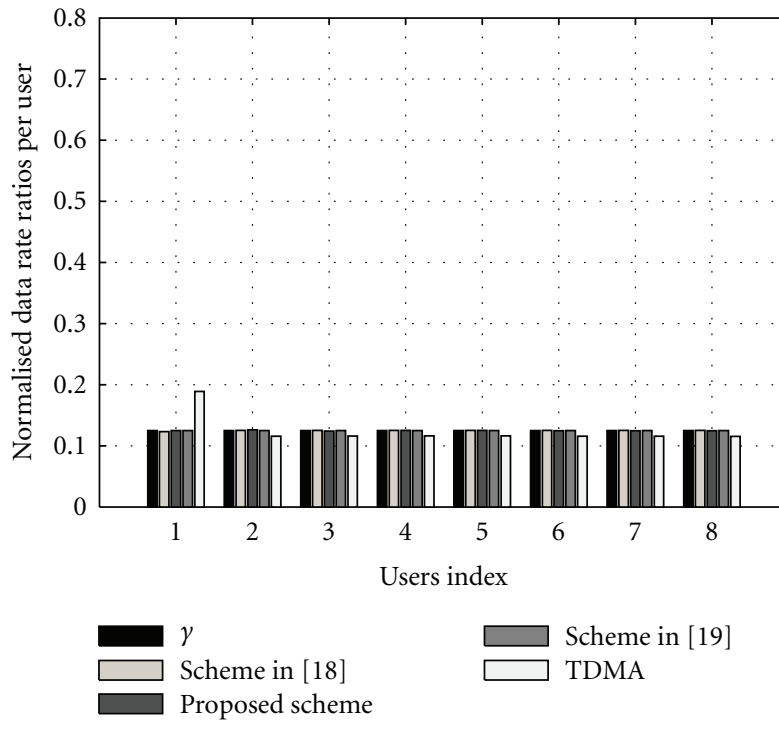

Figure 9: Normalized data rate ratios per user $(p=0)$.

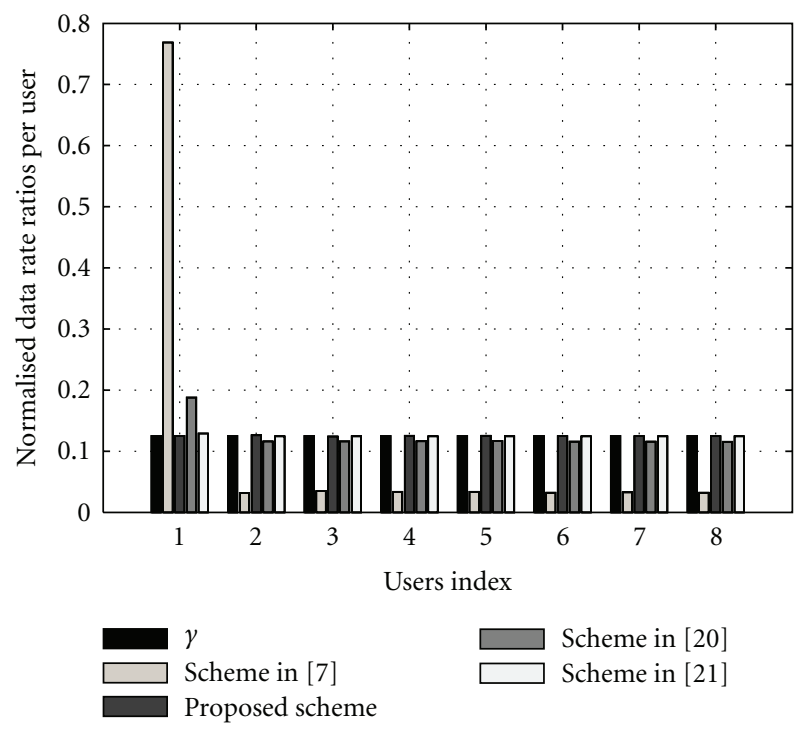

Figure 10: Normalized data rate ratios per user $(p=0)$.

of the users' data rates. The individual normalized data rates of users $2,3, \ldots, 8$ are very little. Static TDMA scheme tends to allocate similar data rates to each user, since all users get the same opportunity to transmit. However, the sum of the users' data rates of the proposed resource allocation scheme is distributed very well, very close to the defined ideal data rate constraints, among users, and equally or better than the other schemes.

\section{Conclusion}

A fairness-aware resource allocation scheme for the SISO downlink over frequency-selective channels was introduced 

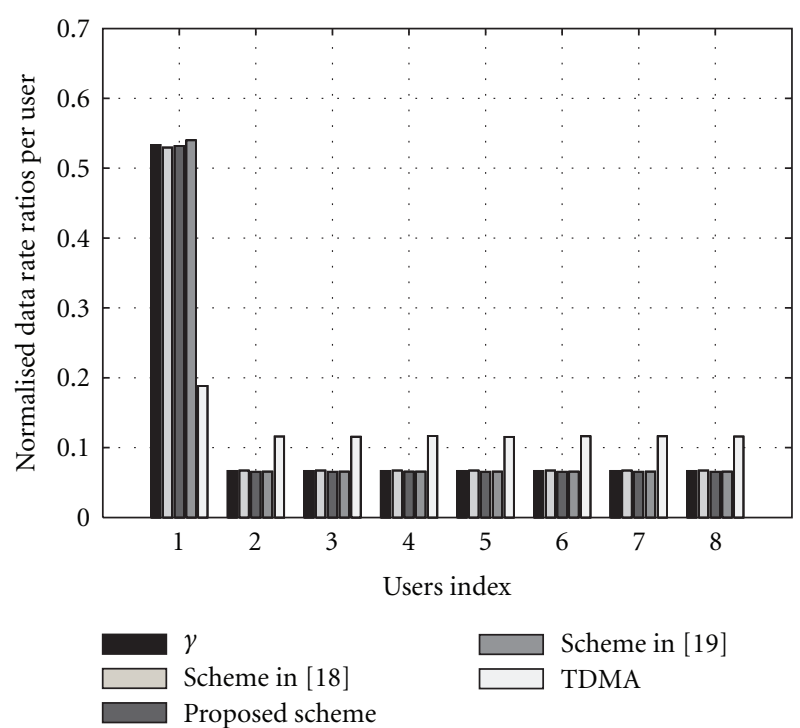

Figure 11: Normalized data rate ratios per user $(p=3)$.
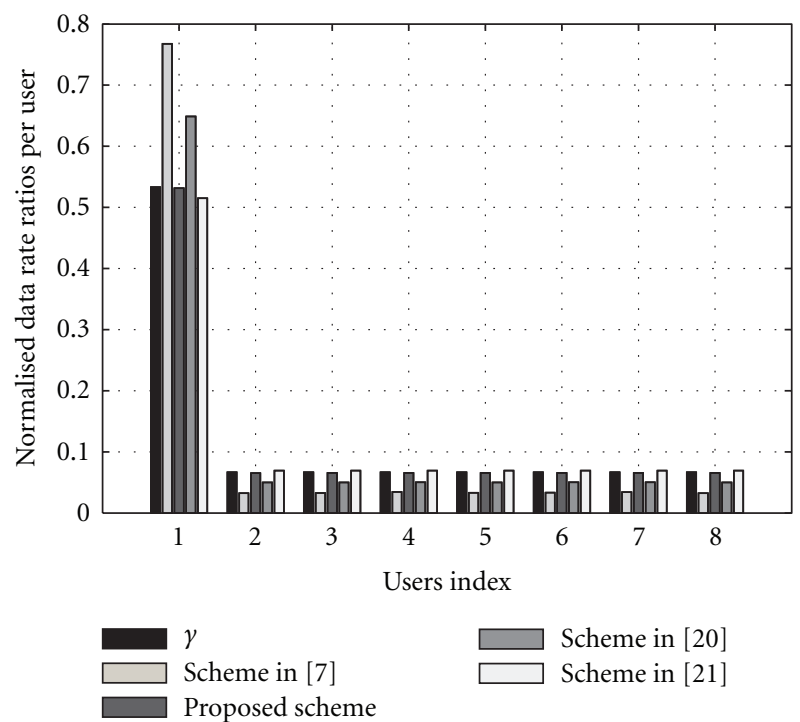

FiguRE 12: Normalized data rate ratios per user $(p=3)$.

which maximizes sum data rate of the system while maintaining rough proportionality among users' data rates. It is also complexity efficient and consists of three algorithms; the first algorithm determines the number of subcarriers to be initially assigned to each user; the second algorithm assigns the subcarriers to each user; and the third one allocates the available power $P_{\text {tot }}$ to subcarriers, using the water-filling equation $[28,29]$ which is optimal. In addition, its innovative priority scheduling exploits more efficiently the multiuser diversity and makes it perform better than previous schemes. Therefore, sum of the users' data rates is distributed more fairly among users, and for different data rate constraints, different proportional data rates can be achieved among users.

\section{References}

[1] H. Sampath, S. Talwar, J. Tellado, V. Erceg, and A. Paulraj, "A fourth-generation MIMO-OFDM broadband wireless system: design, performance, and field trial results," IEEE Communications Magazine, vol. 40, no. 9, pp. 143-149, 2002.

[2] R. V. Nee and R. Prasad, OFDM for Wireless Multimedia Communications, Artech House, Norwood, Mass, USA, 2000.

[3] J. G. Andrews, A. Ghosh, and R. Muhamed, Fundamentals of WiMAX: Understanding Broadband Wireless Networking, Prentice Hall, Upper Saddle River, NJ, USA, 2007.

[4] E. Lawrey, "Multiuser OFDM," in Proceedings of the IEEE International Symposium on Signal Processing and Its Applications (ISSPA '99), vol. 2, pp. 761-764, Brisbane, Australia, August 1999.

[5] D. Tse and P. Viswanath, Fundamentals of Wireless Communication, Cambridge University Press, Cambridge, UK, 2005.

[6] R. Knopp and P. A. Humblet, "Information capacity and power control in single-cell multiuser communications," in Proceedings of the IEEE International Conference on Communications, pp. 331-335, Seattle, Wash, USA, June 1995.

[7] J. Jang and K. B. Lee, "Transmit power adaptation for multiuser OFDM systems," IEEE Journal on Selected Areas in Communications, vol. 21, no. 2, pp. 171-178, 2003.

[8] G. Li and H. Liu, "On the optimality of the OFDMA network," IEEE Communications Letters, vol. 9, no. 5, pp. 438-440, 2005.

[9] S. Boyd and L. Vandenberghe, Convex Optimization, Cambridge University Press, Cambridge, UK, 2004.

[10] H. Rohling and R. Gruenheid, "Performance comparison of different multiple access schemes for the downlink of an OFDM communication system," in Proceedings of the 47th IEEE Vehicular Technology Conference (VTC '97), pp. 13651369, Phoenix, Fla, USA, May 1997.

[11] C. Y. Wong, R. S. Cheng, K. B. Letaief, and R. D. Murch, "Multiuser OFDM with adaptive subcarrier, bit, and power allocation," IEEE Journal on Selected Areas in Communications, vol. 17, no. 10, pp. 1747-1758, 1999.

[12] G. Zhang, "Subcarrier and bit allocation for real-time services in multiuser OFDM systems," in Proceedings of the IEEE International Conference on Communications, pp. 2985-2989, Paris, France, June 2004.

[13] G. Yu, Z. Zhang, Y. Chen, J. Shi, and P. Qiu, "A novel resource allocation algorithm for real-time services in multiuser OFDM systems," in Proceedings of the 63rd IEEE Vehicular Technology Conference (VTC '06), pp. 1156-1160, Melbourne, Australia, May 2006.

[14] D. Kivanc, G. Li, and H. Liu, "Computationally efficient bandwidth allocation and power control for OFDMA," IEEE Transactions on Wireless Communications, vol. 2, no. 6, pp. 1150-1158, 2003.

[15] C. Y. Wong, C. Y. Tsui, R. S. Cheng, and K. B. Letaief, "A real-time sub-carrier allocation scheme for multiple access downlink OFDM transmission," in Proceedings of the 50th IEEE Vehicular Technology Conference (VTC '99), vol. 2, pp. 1124-1128, Amsterdam, The Netherlands, September 1999.

[16] Q. Wang, J. Xu, and Z. Bu, "Proportional-fair bit and power adaptation in multiuser OFDM systems," in Proceedings of the 17th IEEE International Symposium on Personal, Indoor and Mobile Radio Communications, pp. 1-4, Helsinki, Finland, September 2006.

[17] W. Rhee and J. M. Cioffi, "Increase in capacity of multiuser OFDM system using dynamic subchannel allocation," in Proceedings of the 51st IEEE Vehicular Technology Conference (VTC'00), pp. 1085-1089, Tokyo, Japan, May 2000. 
[18] Z. Shen, J. G. Andrews, and B. L. Evans, "Adaptive resource allocation in multiuser OFDM systems with proportional rate constraints," IEEE Transactions on Wireless Communications, vol. 4, no. 6, pp. 2726-2736, 2005.

[19] I. C. Wong, Z. Shen, B. L. Evans, and J. G. Andrews, "A low complexity algorithm for proportional resource allocation in OFDMA systems," in Proceedings of IEEE Workshop on Signal Processing Systems Design and Implementation, pp. 1-6, Austin, Tex, USA, October 2004.

[20] S. Sadr, A. Anpalagan, and K. Raahemifar, "Suboptimal rate adaptive resource allocation for downlink OFDMA systems," International Journal of Vehicular Technology, vol. 2009, Article ID 891367, 10 pages, 2009.

[21] C. Mohanram and S. Bhashyam, "A sub-optimal joint subcarrier and power allocation algorithm for multiuser OFDM," IEEE Communications Letters, vol. 9, no. 8, pp. 685-687, 2005.

[22] Y. J. Zhang and K. B. Letaief, "Multiuser adaptive subcarrierand-bit allocation with adaptive cell selection for OFDM systems," IEEE Transactions on Wireless Communications, vol. 3, no. 5, pp. 1566-1575, 2004.

[23] Z. Mao and X. Wang, "Efficient optimal and suboptimal radio resource allocation in OFDMA system," IEEE Transactions on Wireless Communications, vol. 7, no. 2, Article ID 4450806, pp. 440-445, 2008.

[24] K. A. D. Teo, Y. Otani, and S. Ohno, "Adaptive subcarrier allocation for multi-user OFDM system," IEICE Transactions on Fundamentals of Electronics, Communications and Computer Sciences, vol. E89-A, no. 11, pp. 3131-3137, 2006.

[25] Y. Ma, "Rate maximization for downlink OFDMA with proportional fairness," IEEE Transactions on Vehicular Technology, vol. 57, no. 5, pp. 3267-3274, 2008.

[26] A. Biagioni, R. Fantacci, D. Marabissi, and D. Tarchi, "Adaptive subcarrier allocation schemes for wireless OFDMA systems in wimax networks," IEEE Journal on Selected Areas in Communications, vol. 27, no. 2, Article ID 4769396, pp. 217 225, 2009.

[27] I. Kim, H. L. Lee, B. Kim, and Y. H. Lee, "On the use of linear programming for dynamic subchannel and bit allocation in multiuser OFDM," in Proceedings of the IEEE Global Telecommunications Conference (GLOBECOM '01), pp. 3648-3652, San Antonio, Calif, USA, November 2001.

[28] R. G. Gallager, Information Theory and Reliable Communication, John Wiley \& Sons, New York, NY, USA, 1968.

[29] T. M. Cover and J. A. Thomas, Elements of Information theory, John Wiley \& Sons, Hoboken, NJ, USA, 2006.

[30] A. J. Goldsmith, "Variable-rate variable-power MQAM for fading channels," IEEE Transactions on Communications, vol. 45, no. 10, pp. 1218-1230, 1997.

[31] S. T. Chung and A. J. Goldsmith, "Degrees of freedom in adaptive modulation: a unified view," IEEE Transactions on Communications, vol. 49, no. 9, pp. 1561-1571, 2001.

[32] T. Ibaraki and N. Katoh, Resource Allocation ProblemsAlgorithmic Approaches, M. Garey, Ed., MIT Press, 1988.

[33] W. C. Jakes Jr., Microwave Mobile Communications, John Wiley \& Sons, New York, NY, USA, 1994. 

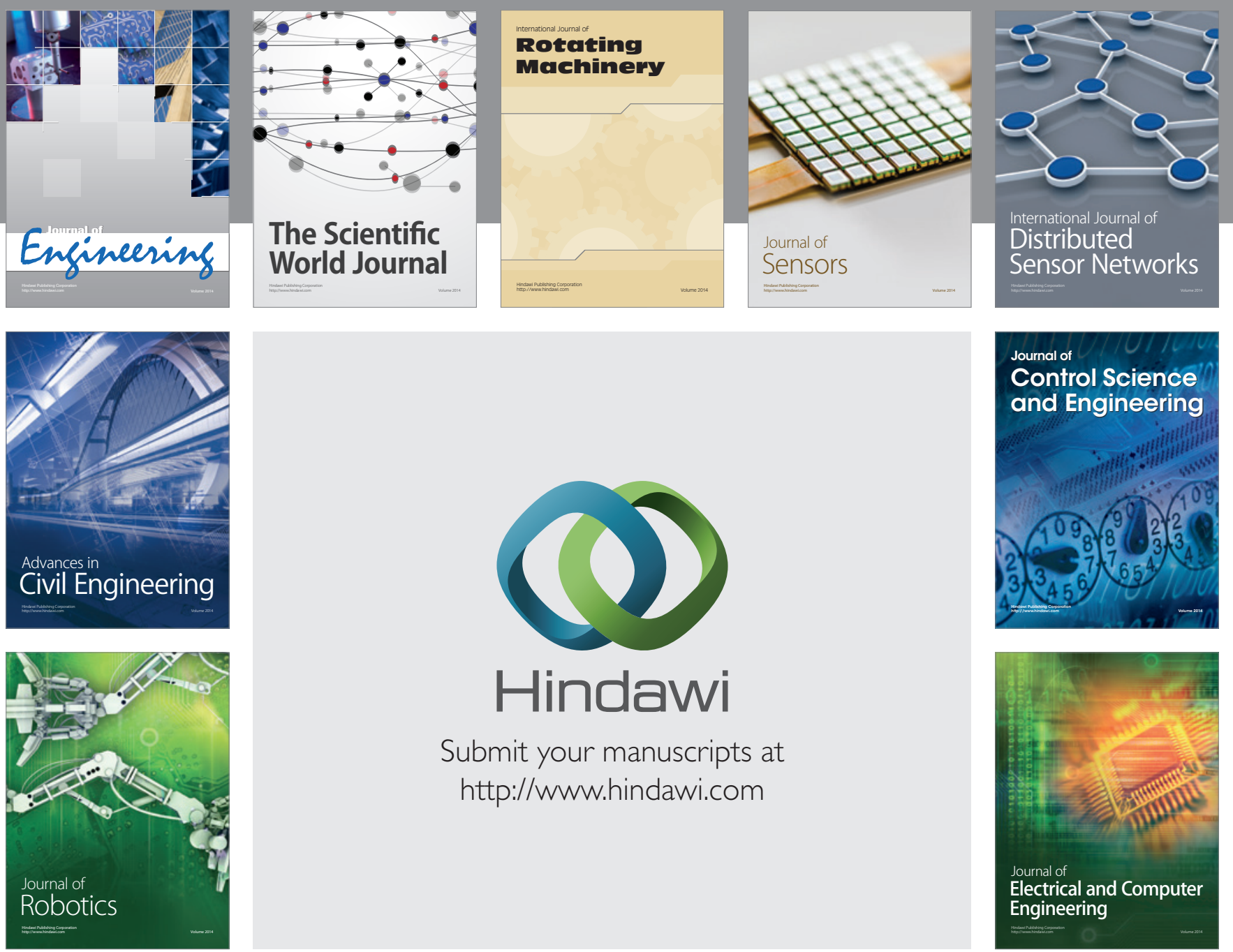

Submit your manuscripts at

http://www.hindawi.com
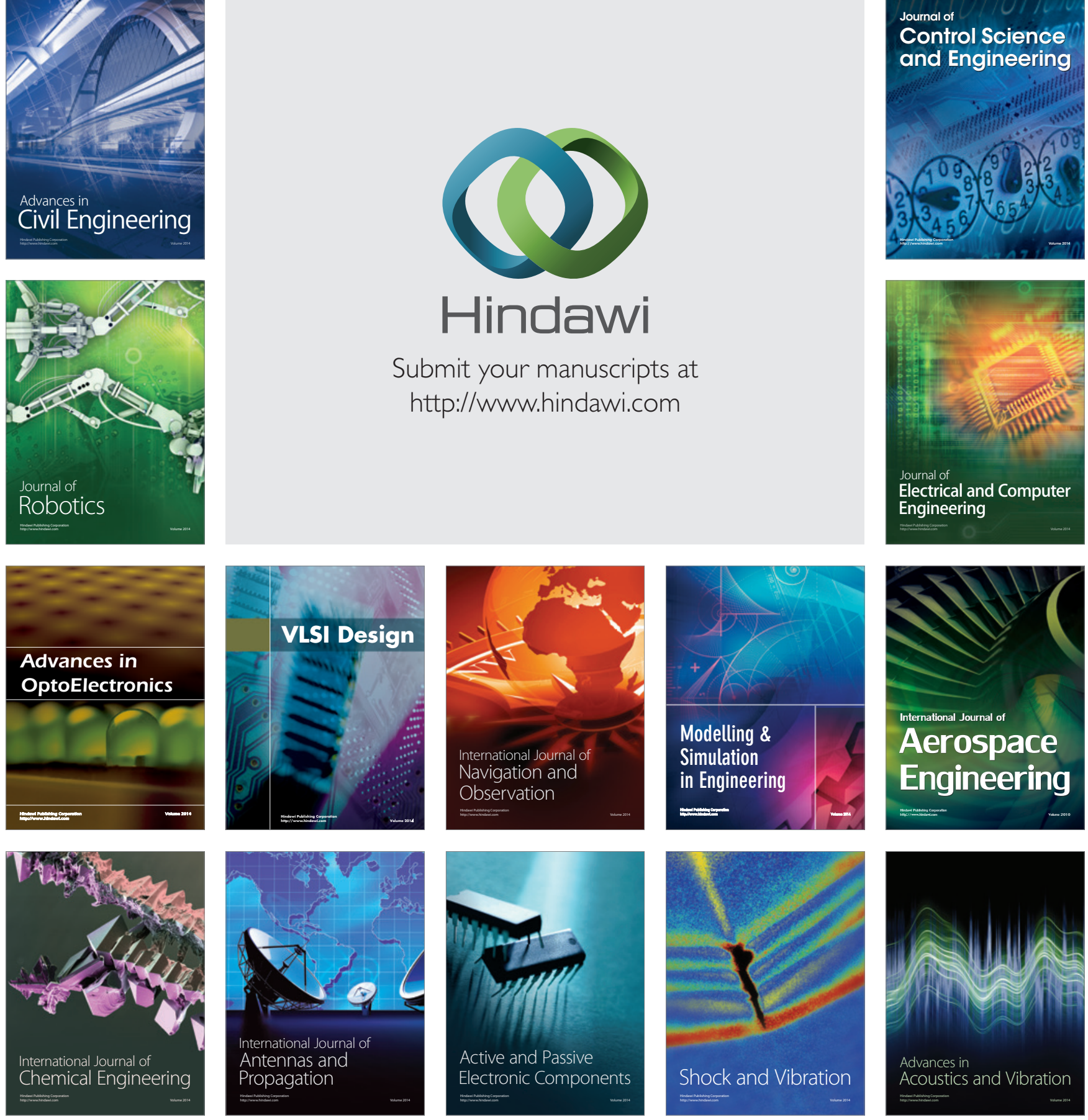